\title{
Multiple Control of Absence Seizures in the Brain: A Computational Study
}

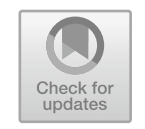

\author{
Daqing Guo
}

\begin{abstract}
As a typical generalized epilepsy, absence epilepsy can be easily identified during absence seizures (AS) based on the electrophysiological hallmark that synchronous 2-4 Hz spike and wave discharges (SWDs) widely observed in the clinical electroencephalography (EEG). Inspired by animal experimental studies that AS may be regulated by neural circuits within and outside the corticothalamic (CT) network, we employed computational models to further dissect the underlying biophysical mechanisms. Simulations show that in the CT network, enhancing the thalamic feedforward inhibition can effectively abate SWDs. Interestingly, the inhibitions mediated by $\mathrm{GABA}_{\mathrm{A}}$ and $\mathrm{GABA}_{\mathrm{B}}$ in the pathway from the thalamic reticular nucleus (TRN) to the specific relay nuclei of thalamus (SRN) play different roles in controlling AS. Specifically, unlike $\mathrm{GABA}_{\mathrm{B}}$ inhibition, $\mathrm{GABA}_{\mathrm{A}}$ inhibition has more influences on the dominant frequency of neural oscillations. On the other hand, the basal ganglia (BG) output pathways from the substantia nigra pars reticulate (SNr) to the TRN and SRN have been demonstrated to contribute to suppressing AS. Note that the control mechanism underlying the SNr-TRN pathway is mainly due to the collision in the TRN, whereas the weakened $\mathrm{GABA}_{\mathrm{B}}$ inhibition is responsible for the suppression of AS via the SNr-SRN pathway. More importantly, the competition between the two output pathways can induce BG bidirectionally controlling AS. Besides these observations, we further found that AS can also be terminated by the newly identified inhibitory pallido-cotrical pathway in our model. These findings highlight the neural circuits that have close associations with the CT network, including internal and external pathways, may have functional roles in regulating AS and may provide a novel insight into the treatment of absence epilepsy.
\end{abstract}

D. Guo $(\varangle)$

MOE Key Laboratory for NeuroInformation, School of Life Science and Technology,

The Clinical Hospital of Chengdu Brain Science Institute, University of Electronic Science and Technology of China, Chengdu 610054, China

e-mail: dqguo@uestc.edu.cn 